\title{
BILATERAL THALAMIC HAEMORRHAGE FOLLOWING BURR HOLE DRAINAGE OF CHRONIC SUBDURAL HEMATOMA
}

Vinayak V. Raje1, Shivaji V. Raje², Pandurang Barve ${ }^{3}$, Devdutta Patil4, Roshan Chiranjeev 5 , Bhushan Shinde 6

1 Associate Professor, Department of Neurosurgery, KIMS Karad.

${ }_{2}^{2}$ MS, Department of Obstetrics and Gynaecology, KIMS Karad.

${ }^{3}$ Resident, Department of Neurosurgery, KIMS Karad.

${ }^{4}$ Resident, Department of Neurosurgery, KIMS Karad.

${ }^{5}$ Resident, Department of Neurosurgery, KIMS Karad.

${ }^{6}$ Resident, Department of Neurosurgery, KIMS Karad.

\section{ABSTRACT}

Bilateral thalamic haemorrhage is an unusual complication of burr hole drainage surgery. We present the case of a patient with this rare postoperative complication of bilateral thalamic haemorrhage following burr hole drainage of a chronic subdural hematoma.

\section{KEYWORDS}

Chronic Subdural Hematoma, Burr Hole.

HOW TO CITE THIS ARTICLE: Raje VV, Raje SV, Barve P, et al. Bilateral thalamic haemorrhage following burr hole drainage of chronic subdural hematoma. J. Evolution Med. Dent. Sci. 2016; 5(11):483-484, DOI: 10.14260/jemds/2016/110

\section{INTRODUCTION}

Chronic subdural hematoma is a common clinical entity treated by neurosurgeons with very good cure rates.[1] Recurrence of the hematoma, infection, seizure, cerebral edema, tension pneumocephalus and failure of the brain to reexpand are well-recognized complications following surgery for chronic subdural hematoma. ${ }^{[2]}$ An intracerebral or intraventricular haemorrhage following such an evacuation is very rarely observed (Incidence 0.7-5\%) with devastating consequences.[2,3,4] We report the case of bilateral thalamic haemorrhage after evacuation of a chronic subdural hematoma.

\section{CASE REPORT}

A 65-year-old male presented with complaints of headache since 10 days, right upper limb and lower limb weakness since 2 days. Past history revealed a minor fall with head injury 1 month ago for which no medical consultation was sought. Neurological examination was unremarkable, except for the right side hemiparesis. MRI of the head showed a left frontoparieto-occipital and right parieto-occipital chronic subdural hematoma with $6 \mathrm{~mm}$ midline shift to right [Figure 1]. After baseline investigations which documented a normal coagulation profile, the patient was taken for left parietal burr hole and drainage of the subdural hematoma under MAC (Monitored Anaesthesia Care) with conscious sedation. After making the burr hole, the dura was coagulated with bipolar cautery and incised in a cruciate manner. The subdural collection was allowed to drain without any negative pressure being applied in the subdural cavity. After the subdural hematoma had drained on its own accord (Owing to the pressure differential), the cavity was gently irrigated with normal saline till the returning fluid was clear.

Financial or Other, Competing Interest: None

Submission 18-12-2015, Peer Review 16-01-2016,

Acceptance 23-01-2016, Published 08-02-2016.

Corresponding Author:

Dr. Pandurang Barve,

Room No. 10, IHR hostel,

KIMS, Karad.

E-mail: pandurangexams@gmail.com

DOI:10.14260/jemds/2016/110
The surgery was uneventful and haemostasis was achieved prior to wound closure. The patient's hemodynamic parameters were stable throughout the procedure. Postoperatively, patient showed moderate neurological deterioration and was not improving as per expectation. So MRI of brain was repeated (Figure 2), which was suggestive of bilateral thalamic haemorrhage. Patient show progressive neurological improvement after $7^{\text {th }}$ post-operative day. At the time of discharge, GCS was 14/15.

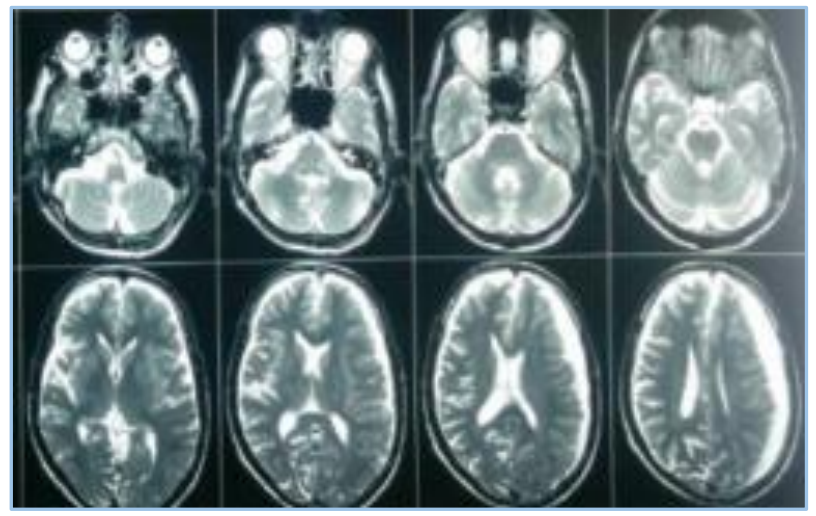

Fig. 1: MRI of the head showed a left frontoparietoccipital and right parietoccipital chronic subdural hematoma with $6 \mathrm{~mm}$ midline shift to right

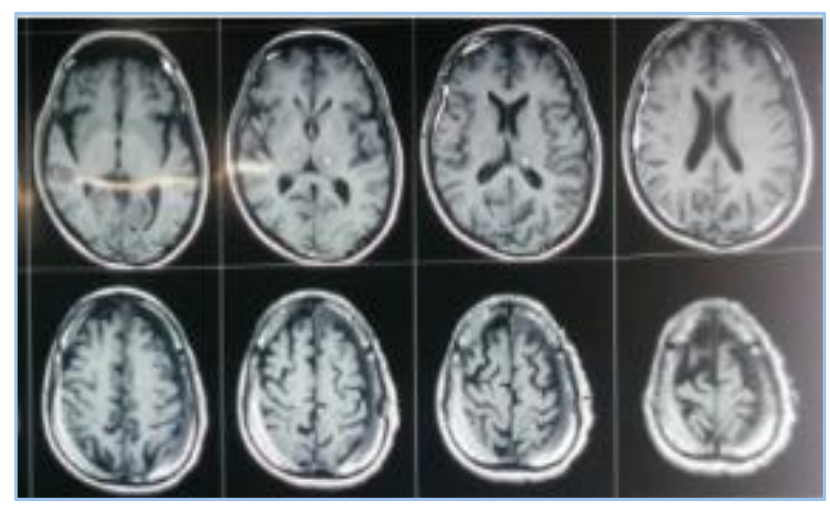

Fig. 2: Post-operative MRI showing bilateral thalamic haemorrhage 


\section{DISCUSSION}

The prognosis of chronic subdural hematoma is relatively good.[1] Some complications do tend to jeopardize the good outcome normally expected in these patients, but none are as devastating as the rare complication of intracerebral haemorrhage (ICH) after evacuation of chronic subdural hematoma.[2,5] Several causative mechanisms have been postulated to explain this complication following a simple surgical procedure. Beneath the chronic subdural hematoma, focal cerebral edema due to impeded venous drainage can reduce cerebral blood flow in the ipsilateral hemisphere. Chronic dilatation of small arterial vessels and loss of carbon dioxide reactivity in the ischemic hemisphere could also contribute to the pathogenesis. Possible pathogenic mechanism for ICH in thus compromised brain include haemorrhage into previously undetected areas of contusion, damage to cerebral vasculature secondary to rapid perioperative parenchymal shift and sudden increase in cerebral blood flow (Post-decompression) combined with focal disruption of auto-regulation. $[2,3]$

Compression by extra-axial collection decreases cerebral blood flow on the affected hemisphere and alters its vascular self-adjustment.[6,7] Some authors hypothesize that the rapid increase in cerebral blood flow (After drainage) in areas of the brain with altered vascular self-adjustment may be the most likely precipitating mechanism of intracerebral haemorrhage after surgical evacuation of chronic subdural hematoma.[2] This mechanism could have resulted in the bilateral thalamic haemorrhage in our patient. However, as the compressed brain is more toward the surface, this mechanism is likely to explain superficial or lobar hematomas. Our case exhibited a bilateral thalamic haemorrhage post-drainage. We hypothesize that after sudden decompression of the brain, the differential expansile qualities of the solid (Brain) and liquid (CSF) components of the cranium may result in mechanical stress at the interface, which in turn may cause rupture of the engorged veins (Due to prolonged compression). This may explain the post-operative MRI finding in our case.

Chronic SDH being a benign entity, neurosurgical outcome have to be optimized for this entity. Considering that intracerebral haemorrhage inadvertently portends a poor outcome for the affected patients, preventive steps are imperative. [2,3]
These include gradual and graded decompression of the chronic subdural haematoma (Especially for large collections) and careful control of blood pressure liability in the perioperative period. The gradual and graded decompression can be achieved by covering the dural aperture with a cottonoid after draining around 15 to $20 \mathrm{~mL}$ of the subdural hematoma. After waiting for approximately $5 \mathrm{~min}$, the cottonoid can be removed to allow further drainage of 15 to $20 \mathrm{~mL}$. This procedure can be continued till the subdural pressures equalize with the atmospheric pressures and at this juncture saline washes can be instituted. This especially holds true for very large subdural hematomas, presenting with raised intra-cranial pressure. Our patient was fortunate to have a good outcome as the bleed was moderate in size; however, we advocate taking steps to prevent such complications altogether.

\section{CONCLUSION}

Bilateral thalamic haemorrhage following burr hole drainage of chronic subdural hematoma is rarity and should be considered in patients who are not improving with burr hole drainage.

\section{REFERENCES}

1. Mori K, Maeda M. Surgical treatment of chronic subdural hematoma in 500 consecutive cases: clinical characteristics, surgical outcome, complications and recurrence rate. Neurol Med Chir (Tokyo) 2001;41:37181.

2. D'Avella D, De Blasi F, Rotilio A, et al. Intracerebral hematoma following evacuation of chronic subdural hematomas. Report of two cases. J Neurosurg 1986; 65:710-2.

3. Muneza S, Rasoloherimampiononiaina MR, Nduwamariya MJ. Postoperative intracerebral and intraventricular hemorrhages following removal of a chronic subdural hematoma. J Clin Neurosci 2009;16:1346-8.

4. Ulivieri S, Oliveri G. Intracerebral haemorrhage following surgical evacuation of chronic subdural haematoma: case report. G Chir 2008;29:233-4.

5. Sousa J, Golash A, Vaz J, et al. Spontaneous intracerebral haemorrhage following evacuation of chronic subdural hematomas. J Clin Neurosci 2004;11:794-6. 